\title{
A HIGH-RESOLUTION GAMMA-RAY SPECTROSCOPIC STUDY OF THE DECAY ${ }^{77} \mathrm{Ge} \rightarrow{ }^{77}$ As
}

\author{
D. P. DONNELLY, J. J. REIDY and M. L. WIEDENBECK \\ Department of Physics, University of Michigan, Ann Arbor, Michigan, USA ${ }^{\dagger}$
}

Received 29 January 1968

\begin{abstract}
The energy and relative intensity values of 82 gamma rays following the decay of ${ }^{77} \mathrm{Ge}$ have been measured. The energy values of seven of these lines were measured using a $2 \mathrm{~m}$ curvedcrystal spectrometer; the other measurements were made using $\mathrm{Ge}(\mathrm{Li})$ spectrometers. A level scheme was constructed which included 28 excited levels and places 74 transitions. The energies of the levels (in $\mathrm{keV}$ ) are 215.5, 264.5, 459.6, 461.4, 475.5, 613.6, 632.1, 784.8, 875.3, 914.2, $1189.7,1202.7,1280.8,1319.6,1382.5,1398.4,1457.7,1479.9,1539.6,1560.6,1717.4,1719.7$, 2000.2, 2109.0, 2143.7, 2303.5, 2341.7 and 2513.2.
\end{abstract}

RADIOACTIVITY: ${ }^{77} \mathrm{Ge}$ [from ${ }^{76} \mathrm{Ge}(\mathrm{n}, \gamma)$ ]; measured $E_{\gamma}, I_{\gamma} \cdot{ }^{77} \mathrm{As}$ deduced levels. Enriched targets, $\mathrm{Ge}(\mathrm{Li})$ detector, curved crystal spectrometer.

\section{Introduction}

The decay of $11.3 \mathrm{~h}{ }^{77} \mathrm{Ge}$ to levels in ${ }^{77}$ As has been studied by several investigators [refs. ${ }^{1-7}$ )]. The gamma-ray spectrum associated with this decay is known to be quite complex. Therefore it appeared desirable to investigate this spectrum with $\mathrm{Ge}(\mathrm{Li})$ spectrometers.

\section{Experimental procedure}

\subsection{THE Ge(Li) SPECTROMETER}

The $\mathrm{Ge}(\mathrm{Li})$ spectrometers used in the present energy and relative intensity measurements included two planar detectors having active volumes $4 \mathrm{~cm}^{2} \times 0.5 \mathrm{~cm}$ and $8 \mathrm{~cm}^{2} \times 0.5 \mathrm{~cm}$ and a $17 \mathrm{~cm}^{3}$ coaxial detector. The associated electronics included either a Tennelec TC-130 or Ortec 118A preamplifier, a Tennelec TC-200 amplifier and a TC-250 biased amplifier and a Victoreen SCIPP 1600 multi-channel pulseheight analyser. The TC-130 and TC-200 units were modified to include pole-zero cancellation.

\subsection{SOURCE}

The sources used in this study were prepared from $\mathrm{Ge}$ metal, natural $\mathrm{GeO}_{2}$ and samples of $\mathrm{GeO}_{2}$ enriched in ${ }^{76} \mathrm{Ge}$. The samples were encapsulated in quartz and irradiated in the Ford Nuclear reactor at a flux of $1-2 \times 10^{13}$ neutrons $/ \mathrm{cm}^{2} \cdot \mathrm{sec}$ for

† Work supported in part by the U.S. Atomic Energy Commission. 
periods ranging from 2 to $24 \mathrm{~h}$. The $\mathrm{GeO}_{2}$ samples were irradiated with a small amount of water; after irradiation the mixture was placed in a lucite source holder, allowed to evaporate and covered with Scotch tape.

\section{Results}

\subsection{ENERGY MEASUREMENTS}

The gamma-ray spectrum in the energy range from $150-2500 \mathrm{keV}$ is shown in figs. 1 and 2. Energy and relative intensity values for 82 gamma rays are presented in table 1.

The specttometers were calibrated for energy and relative intensity measurements using the methods described in ref. ${ }^{8}$ ). This method used as the definition of a peak area $A=\sum_{i}\left(N_{i}-B_{i}\right)$, where $N_{i}$ are the actual number of counts in channel $i$ and $B_{i}$ the background counts in that channel $\left(B_{i}\right.$ is defined by the straight line separating the peak from the background distribution).

The centroid of the area $A$ was used to define the position of a peak in energy determinations. The non-linearities of the detecting system were determined using a precision pulser ${ }^{8}$ ). The energy calibration was obtained by a least-squares fit to the internal calibration lines. The errors include uncertainties in the energies of the calibration lines, peak positions and electronic system non-linearities.

The internal calibration lines had energies $264.45 \pm 0.025,367.49 \pm 0.04,416.35 \pm$ $0.04,557.7 \pm 0.08,714.1 \pm 0.09,810.5 \pm 0.2,1085.0 \pm 0.15$ and $1193.3 \pm 0.2 \mathrm{keV}$. The energy values of the five lines from $264-714 \mathrm{keV}$ were measured with the University of Michigan $2 \mathrm{~m}$ curved crystal spectrometer (CCS). The energy values of the three remaining calibration lines were determined from a spectrum which was obtained by detecting simultaneously the gamma rays following the decay of ${ }^{77} \mathrm{Ge}$ and ${ }^{46} \mathrm{Sc}$. The calibration lines measured with the CCS and the ${ }^{46} \mathrm{Sc}$ lines were used for the energy calibration. For the measurement of higher energy lines, an external calibration line (following the decay of ${ }^{24} \mathrm{Na}$ ) at $2753.92 \pm 0.12 \mathrm{keV}$ [ref. ${ }^{9}$ )] was used.

For those lines which are not resolved, a graphical peel-apart technique was employed. The photopeak line shape for a single line in the energy region of interest was successively fitted to the high-energy end of the composite peak (which has had the background subtracted) and subtracted out. A fiducial mark is put at the centroid of the standard line shape which is fitted into the composite and each line in the composite is marked and its centroid is read directly from the graph.

Peel-aparts were performed on gamma-ray lines in the energy regions 747,783 , $925,1310,1470,1720,2080,2245$ and $2340 \mathrm{keV}$. The singles spectrum in the region from 740-940 keV, and the peel-aparts required in that energy region are shown in fig. 3. The spectrum was taken being a biased amplifier; the slope of the calibration line was $0.39 \mathrm{keV} / \mathrm{channel}$. In this energy region, the $810.5 \mathrm{keV}$ line was used as the standard line shape. The group of lines in the $747 \mathrm{keV}$ region appears to be comprised of one weak and two strong lines. A comparison of the curvature of the low-energy 


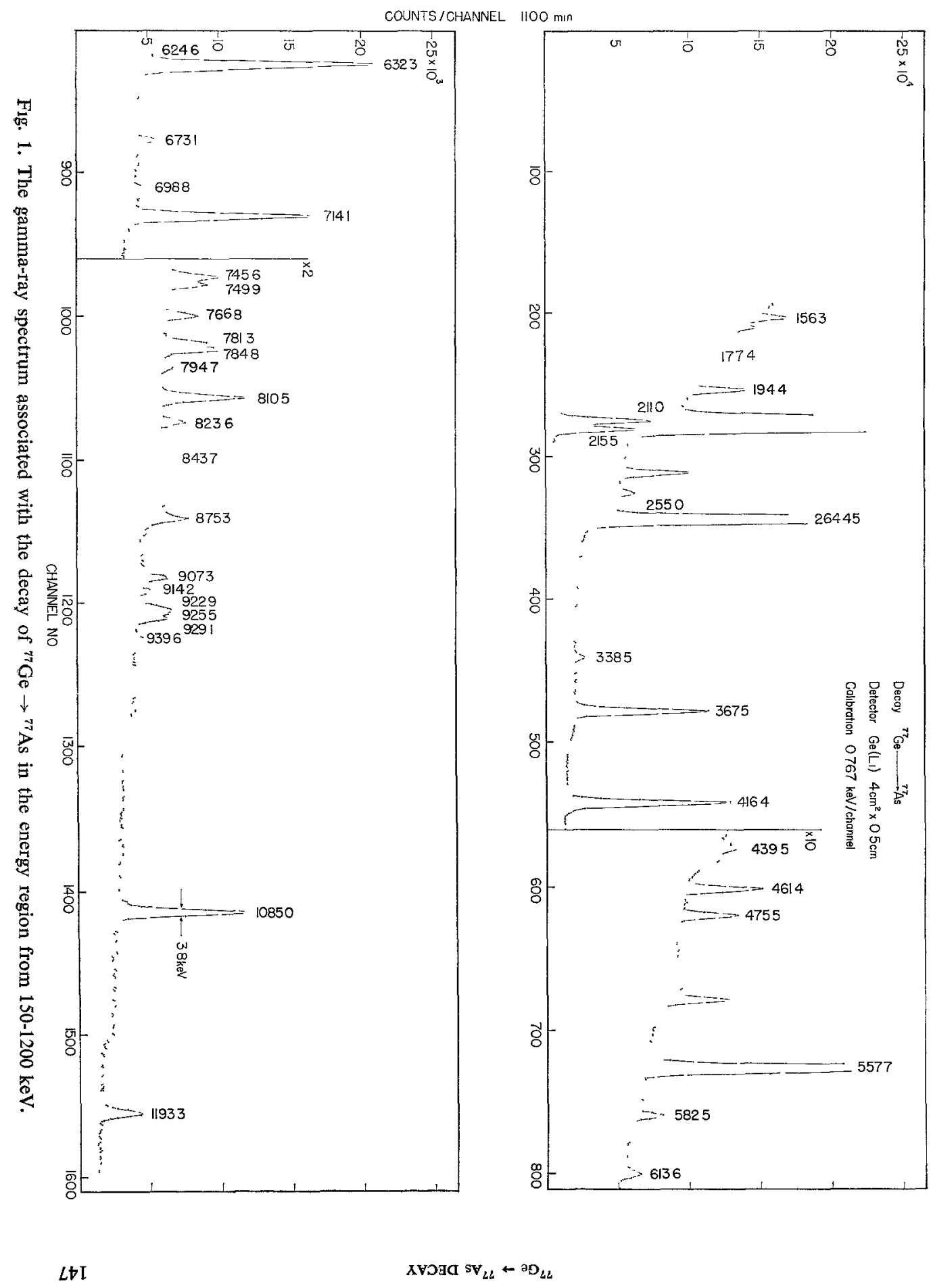




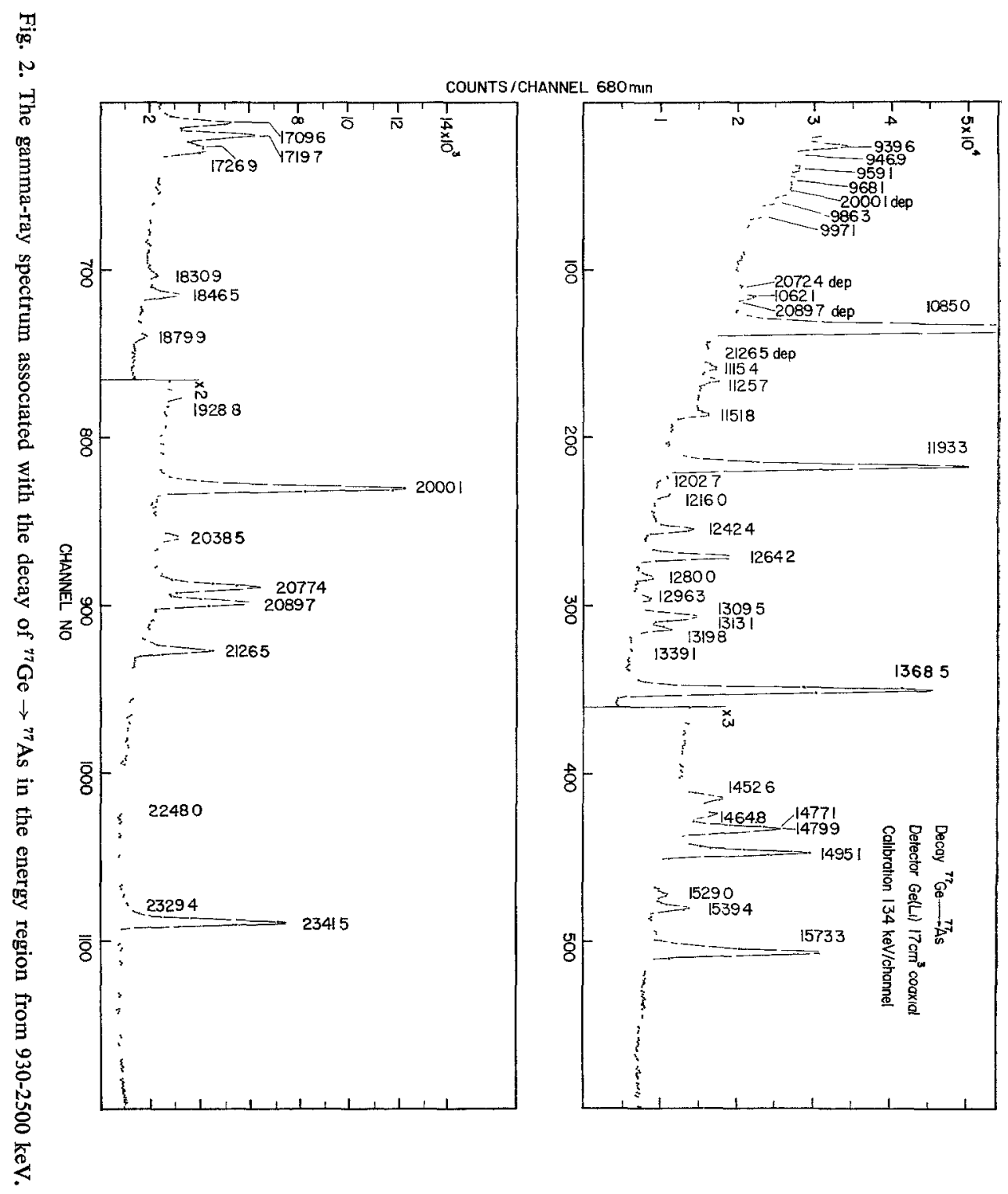




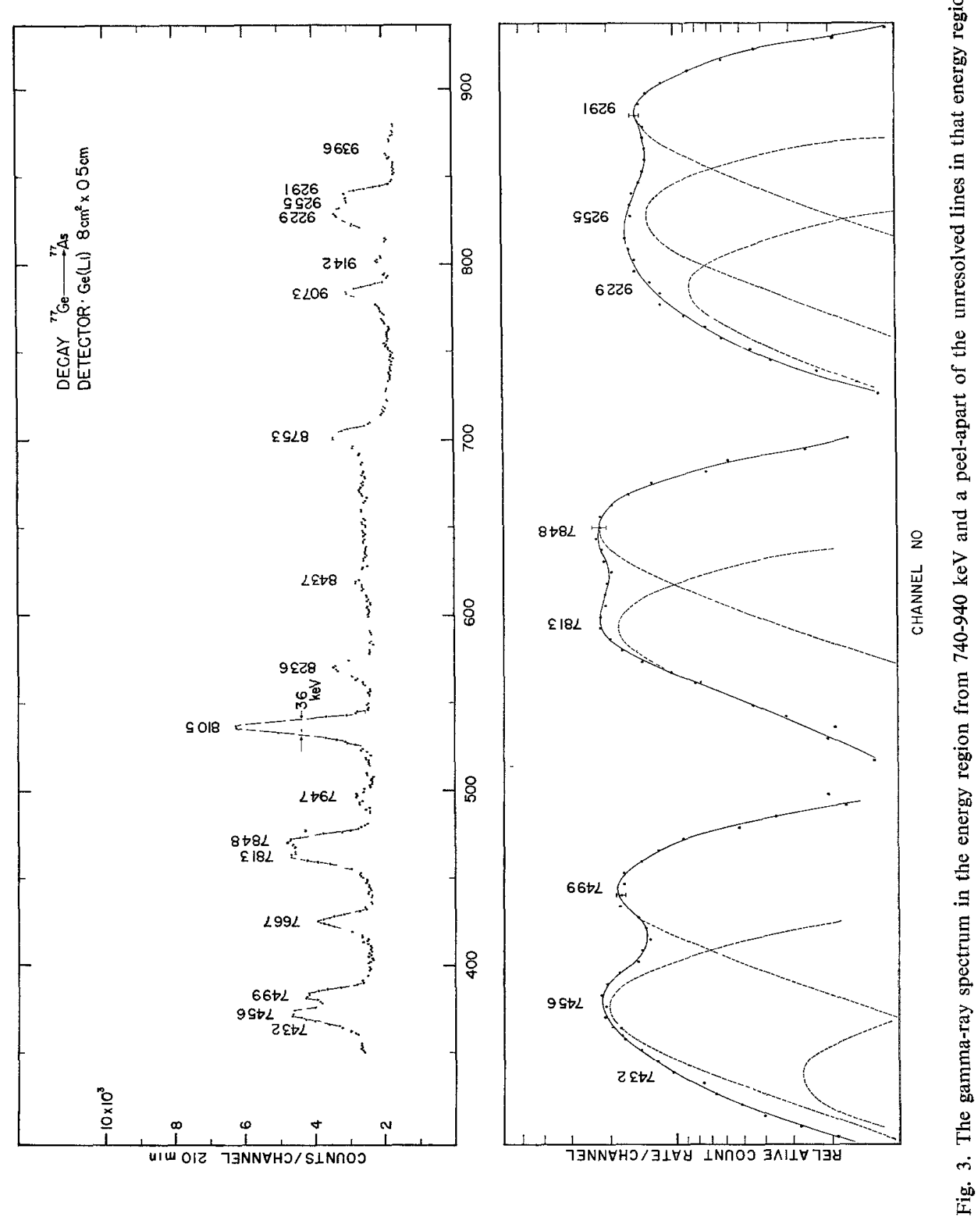


side of the three groups lends support to the presence of a weak line at $743.2 \mathrm{keV}$. Since the line profiles for the other groups in this region gave such good fits, the weak $743 \mathrm{keV}$ line is included in table 1.

TABLE 1

Energy and relative intensity values for gamma rays following the decay of ${ }^{7} \mathrm{Ge}$

\begin{tabular}{|c|c|c|c|}
\hline Energy & Relative intensity & Energy & Relative intensity \\
\hline $156.3 \pm 0.3$ & $16.3 \pm 2.2$ & $986.3 \pm 0.8$ & $2.3 \pm 0.6$ \\
\hline $177.4 \pm 0.4$ & $1.0 \pm$ & $997.1 \pm 0.5$ & $2.3 \pm 0.5$ \\
\hline $194.9 \pm 0.2$ & $48.5 \pm$ & $1062.1 \pm 1.5$ & $1.4 \pm 0.5$ \\
\hline $\left.211.03 \pm 0.04^{\mathrm{a}}\right)$ & $998.0 \pm 90.0$ & $1085.0=0.15$ & $216.0 \pm 14.0$ \\
\hline $\left.215.51 \pm 0.04^{a}\right)$ & $871.0 \pm 78.0$ & $1115.4 \pm 0.4$ & $3.1 \pm 0.6$ \\
\hline $255.0 \pm 0.25$ & $2.6 \pm 2.1$ & $1125.7 \pm 0.4$ & $3.0 \pm 0.6$ \\
\hline $\left.264.45 \pm 0.025^{\mathrm{a}}\right)$ & $1700.0 \pm 116.0$ & $1151.8 \pm 0.4$ & $6.2 \pm 1.4$ \\
\hline $338.5 \pm 0.15$ & $30.8 \pm 2.8$ & $1193.3 \pm 0.2$ & $86.2 \pm 4.3$ \\
\hline $\left.367.49 \pm 0.04^{\mathrm{a}}\right)$ & $485.0 \pm 32.0$ & $1202.7 \pm 0.6$ & $2.0 \pm 1.0$ \\
\hline $\left.416.35 \pm 0.04^{\mathrm{a}}\right)$ & $815.0 \pm 51.0$ & $1216.0 \pm 0.4$ & $3.5 \pm 1.3$ \\
\hline $439.5 \pm 0.23$ & $10.3 \pm 5.1$ & $1242.4 \pm 0.3$ & $10.4 \pm 2.8$ \\
\hline $461.4 \pm 0.22$ & $41.9 \pm$ & $1264.2 \pm 0.3$ & $26.7 \pm 3.2$ \\
\hline $475.5 \pm 0.15$ & $35.9 \pm$ & $1280.8 \pm 0.4$ & $3.4 \pm 0.9$ \\
\hline $\left.557.7 \pm 0.08^{a}\right)$ & $569.0 \pm 28.0$ & $1296.3 \pm 0.4$ & $2.9 \pm 0.7$ \\
\hline $582.5 \pm 0.18$ & $25.5 \pm 3.9$ & $1309.5 \pm 0.5$ & $16.1 \pm 2.9$ \\
\hline $613.6 \pm 0.4$ & $23.9 \pm 5.8$ & $1313.1 \pm 0.5$ & $9.2 \pm 1.7$ \\
\hline $624.6 \pm 0.4$ & $2.0 \pm 0.6$ & $1319.8 \pm 0.5$ & $13.5 \pm 2.2$ \\
\hline $632.3 \pm 0.23$ & $314.0 \pm 16.0$ & $1368.5 \pm 0.5$ & $90.5 \pm 9.1$ \\
\hline $673.1 \pm 0.25$ & $21.9 \pm 1.9$ & $1452.6 \pm 0.7$ & $5.9 \pm 1.4$ \\
\hline $698.8 \pm 0.35$ & $8.35 \pm \quad 0.69$ & $1464.8 \pm 0.8$ & $5.5 \pm 1.3$ \\
\hline $\left.714.1 \pm 0.09^{a}\right)$ & $269.0 \pm 14.0$ & $1477.1 \pm 0.6$ & $11.1 \pm 1.9$ \\
\hline $743.2 \pm 0.40$ & 4.8 土 1.4 & $1479.9 \pm 0.6$ & $8.3 \pm 1.4$ \\
\hline $745.6 \pm 0.28$ & $35.2 \pm$ & $1495.1 \pm 0.5$ & $18.6 \pm 3.0$ \\
\hline $749.9 \pm 0.28$ & $32.6 \pm$ & $1529.0 \pm 0.5$ & $2.1 \pm 0.6$ \\
\hline $766.8 \pm 0.25$ & $26.0 \pm 2.6$ & $1539.4 \pm 0.5$ & $4.9 \pm 1.4$ \\
\hline $781.3 \pm 0.25$ & $33.5 \pm 4.0$ & $1573.3 \pm 0.25$ & $22.7 \pm 1.4$ \\
\hline $784.8 \pm 0.25$ & $40.9 \pm 4.9$ & $1709.6 \pm 0.6$ & $10.4 \pm 2.5$ \\
\hline $794.7 \pm 0.35$ & $4.9 \pm$ & $1719.7 \pm 0.6$ & $14.7 \pm 2.9$ \\
\hline $810.5 \pm 0.20$ & $78.1 \pm$ & $1726.9 \pm 0.9$ & $4.2 \pm 1.3$ \\
\hline $823.6 \pm 0.26$ & $20.9 \pm 3.6$ & $1830.9 \pm 0.8$ & $1.5 \pm 0.5$ \\
\hline $843.7 \pm 0.30$ & $7.9 \pm 2.1$ & $1846.5 \pm 0.5$ & $5.4 \pm 1.6$ \\
\hline $875.3 \pm 0.30$ & $26.0 \pm 10.0$ & $1879.9 \pm 0.8$ & $1.0 \pm 0.4$ \\
\hline $907.3 \pm 0.35$ & $22.3 \pm 4.1$ & $1928.8 \pm 0.8$ & $0.7 \pm 0.3$ \\
\hline $914.2 \pm 0.5$ & $4.2 \pm$ & $2000.1 \pm 0.3$ & $19.2 \pm 3.7$ \\
\hline $922.9 \pm 0.35$ & $16.8 \pm 2.5$ & $2038.5 \pm 0.5$ & $1.2 \pm 0.3$ \\
\hline $925.5 \pm 0.30$ & $26.2 \pm 3.9$ & $2077.4 \pm 0.5$ & $8.0 \pm 2.3$ \\
\hline $929.1 \pm 0.30$ & $29.2 \pm$ & $2089.7 \pm 0.5$ & $7.3 \pm 2.2$ \\
\hline $939.6 \pm 0.35$ & $6.5 \pm$ & $2126.5 \pm 0.4$ & $4.8 \pm 15$ \\
\hline $946.9 \pm 1.0$ & $0.4 \pm \quad 0.25$ & $2248.0 \pm 1.0$ & $0.7 \pm 0.3$ \\
\hline $959.1 \pm 0.8$ & $2.6 \pm 0.7$ & $2329.4 \pm 1.0$ & $0.8 \pm 0.2$ \\
\hline $968.1 \pm 0.8$ & $2.4 \pm \quad 0.6$ & $2341.5 \pm 0.4$ & $16.4 \pm 3.2$ \\
\hline
\end{tabular}

a) Energy measured with curved-crystal spectrometer. 


\subsection{RELATIVE INTENSITY MEASUREMENTS}

The relative intensities of 82 gamma rays following the decay of $11.3 \mathrm{~h}{ }^{77} \mathrm{Ge}$ have been measured. The results are presented in table 1 . The detectors were calibrated for full energy peak detection efficiency using sources with well-measured gamma-ray emission rates; the peak area $A$ was defined as indicated above. The detector calibration procedure and the relative detection efficiency curve for one of the detectors are presented in ref. ${ }^{8}$ ).

A half-life check was made of all the lines. From a set of four or five spectra taken in sequence (and either preceded or followed by a background run), the areas of all the peaks were determined, the areas for each spectrum were normalized to a specified line (which is associated with the decay being studied), and then the normalized area values were compared as a function of time.

In cases where lines are not resolved, e.g., at 745 or $783 \mathrm{keV}$, the half-life check was made of the composite peak and a graphical check was made to see if the composite peak maintained the same shape.

Confidence that the lines belong to the $11.3 \mathrm{~h}$ decay of ${ }^{77} \mathrm{Ge} \rightarrow{ }^{77} \mathrm{As}$ does not depend solely on the half-life check method. The spectrum has been observed on many occasions with radioactive sources prepared from four different $\mathrm{Ge}$ samples; $\mathrm{Ge}$ metal, natural $\mathrm{GeO}_{2}$ and two different samples of $\mathrm{GeO}_{2}$ enriched in ${ }^{76} \mathrm{Ge}$. No line in table 1 has been examined fewer than five times with the exception of the 742 $\mathrm{keV}$ line.

\section{The construction of the level scheme for ${ }^{77} \mathrm{As}$}

The level scheme presented here was constructed with the present energy and intensity measurements. The energy combination principle was used, and a search was måde for sets of levels and transitions whose energies summed to the same value. With a large number of transitions and levels, this is a complicated procedure and a systematic approach is needed. Bäcklin has recently published a paper ${ }^{10}$ ) in which he describes a computer program which searches for new levels using known levels and measured transition energies. The program records for an excitation energy $E$ all transition energies $E_{\mathrm{t}}$ and corresponding known energy levels $E_{1}$ which fulfill the condition

$$
|| E-E_{\mathrm{t}}\left|-E_{1}\right| \leqq \Delta E_{\mathrm{t}}+\Delta E_{\mathrm{l}},
$$

where $\Delta E_{\mathrm{t}}$ and $\Delta E_{1}$ are the experimental errors in $E_{\mathrm{t}}$ and $E_{1}$, respectively. The energy value $E$ is increased in small steps to scan the energy region of interest.

Levels at $215.5,264.5,475.5,632.1,1189.7,1457.7,1560.6,2000.2$ and $2341.7 \mathrm{keV}$ are well established. The transitions between these levels and from these levels to the ground state include the 11 strongest gamma-ray transitions in the decay scheme. Their placement in the level scheme is supported by gamma-gamma coincidence data ${ }^{4,5,7}$ ).

These nine levels were taken as established and their energy values together with the complete list of gamma-ray transition energy values were used as input data for 


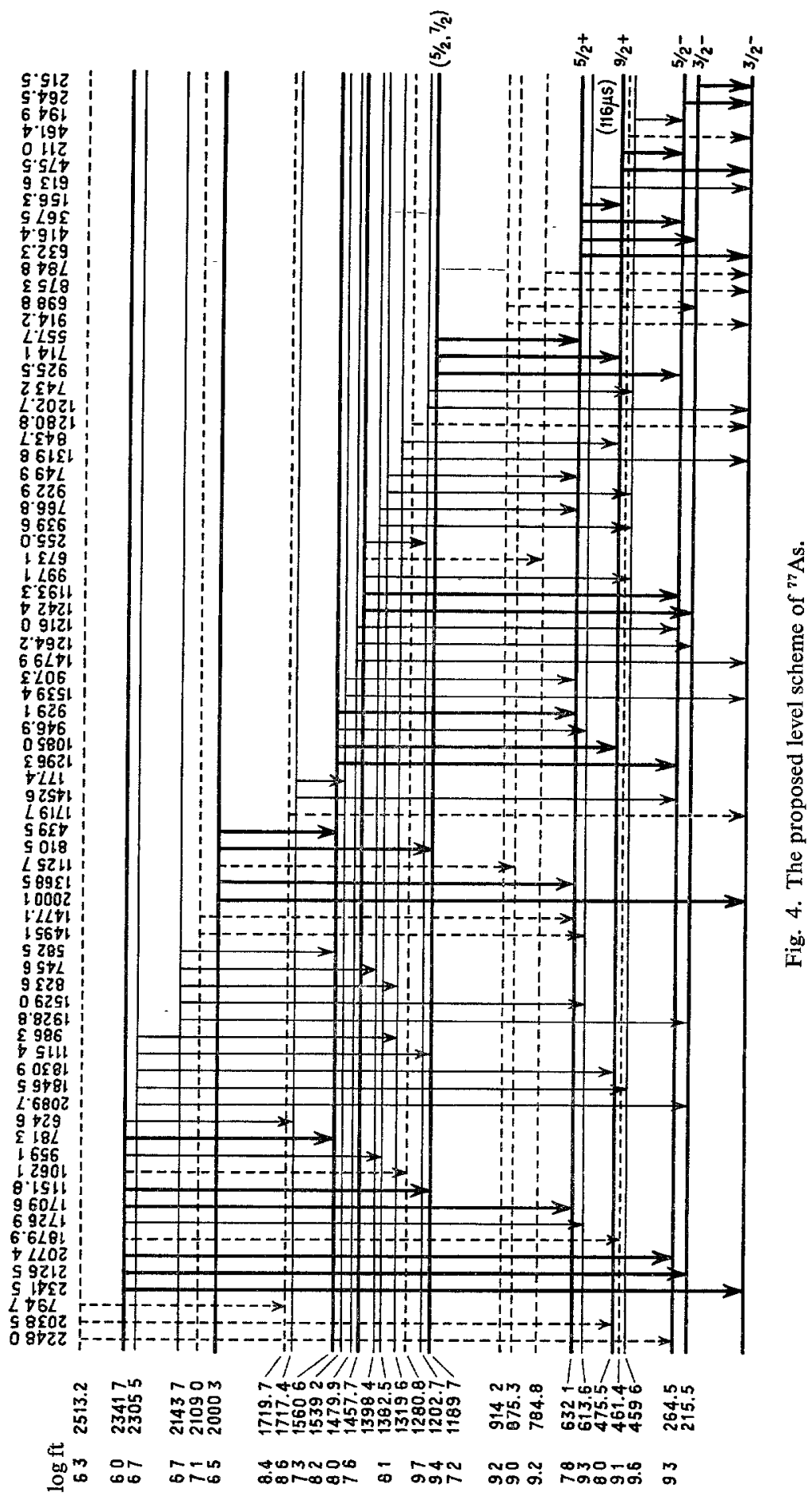


the computer program mentioned above. The entire energy region from $0-2750 \mathrm{keV}$ was scanned in steps of $0.1 \mathrm{keV}$, new levels were found and placed in the known level list, and the program was rerun.

In those cases where a transition could fit between two or more sets of levels, the transitions' placement possibilities were sometimes reduced with the use of relative intensities. In all cases the gamma-ray transition intensity depopulating a level was greater than or equal to the gamma-ray transition intensity feeding the level.

The level scheme presented in fig. 4 has 28 excited levels and places 74 transitions. In the figure, the levels and transitions are presented by lines of different thickness, the thickness being related to the degree of confidence in the level. The nine levels already listed above are placed in category (1); most probable (heavy lines). None of the new levels have the "strength" of these nine established levels. The strength is an indication of the total relative gamma-ray transition intensity associated with the feeding and depopulating of the level, where particular emphasis is placed on the intensity of gamma-ray transitions feeding the level from levels of category (1) and depopulating the level to levels of category (1). For the intermediate category (2) (thin lines), the minimum condition for including a level is the placement of three additional transitions in the level scheme. Less probable levels (3) (dotted lines) were postulated, where each of these levels would place two of the remaining transitions. In fig. 4, the transitions are distinguished just as are the levels, bearing the signature of the lower category of the two levels they connect. The energies of the levels (in keV) are listed by category (as indicated above)

(1) $215.5,264.5,475.5,632.1,1189.7,1560.6,2000.2,2341.7$;

(2) $459.6,613.6,1202.7,1319.6,1382.5,1398.4,1479.9,1539.6,1717.4,2143.7$, 2305.5

(3) $461.4,784.8,875.3,914.2,1280.8,1719.7,2109.0,2513.2$.

The nine levels of the most probable category (1) are close in energy to the values given in ref. ${ }^{7}$ ). The agreement between transitions feeding and depopulating these levels in the present work and those of van der Kooi and van den Bold ${ }^{7}$ ) is quite good. The correspondence, however, between the 11 levels of the present work in category (2) and levels in ref. ${ }^{7}$ ) is very slight. Only the level at $1717.4 \mathrm{keV}$ has a possible correspondence with the $1700 \mathrm{keV}$ level in the decay scheme of ref. ${ }^{7}$ ). All the other proposed levels in category (2) are new.

In the least probable category, eight levels are listed. Three of these levels (at 784.8, 914.2 and $1280.8 \mathrm{keV}$ ) may correspond to levels (at 755, 925 and $1280 \mathrm{keV}$ ) in the decay scheme of van der Kooi and van den Bold ${ }^{7}$ ). Levels at 2232 and $2565 \mathrm{keV}$ in ref. ${ }^{7}$ ) seem to have little support from present energy sums. Although there is a level at $2109 \mathrm{keV}$ both in ref. ${ }^{7}$ ) and in the present work, there is no correspondence between the transitions depopulating the level.

Log $f t$ values determined using the present gamma-ray transition intensity values are presented in fig. 4. The shape factor was assumed to be constant; the beta feeding. to the ground state was assumed to be zero. 


\section{Discussion}

Spin assignments to levels in ${ }^{77}$ As cannot be made at present strictly on the basis of experimental data. However, from measurements of $\log f t$ values, gamma-ray transition rates and gamma-gamma angular correlations, and through analogy with the level schemes of ${ }^{73,75} \mathrm{As}$, the spins of several low-lying levels in ${ }^{77}$ As have been tentatively assigned ${ }^{4,6,7}$ ).

In recent work Robinson, McGowan, Stelson and Milner ${ }^{11}$ ) discussed and summarized their data which dealt with the low-lying levels of odd-mass medium-weight nuclei and indicated features common to these nuclei. For example, they noted that only one spin $\frac{7}{2}$ state is excited through Coulomb excitation and the energy of the level is between 1.1 and 1.4 times the energy of the $2^{+}$state in the neighboring evenmass nucleus. If a $\frac{7}{2}$ level were present in ${ }^{77} \mathrm{As}$, then according to the above it should lie between 620 and $790 \mathrm{keV}$. There are two proposed levels $(613.6$ and $784.8 \mathrm{keV}$ ) in this energy region either of which might be the $\frac{7}{2}$ level.

It was also pointed out ${ }^{11}$ ) that in the level schemes of ${ }^{75} \mathrm{As}$ and ${ }^{79} \mathrm{Br}$ there is a triplet of low-lying levels (between 198 and $280 \mathrm{keV}$ in ${ }^{75} \mathrm{As}$ and between 217 and 307 $\mathrm{keV}$ in ${ }^{79} \mathrm{Br}$ ) with probable spins $\frac{1}{2}, \frac{3}{2}$ and $\frac{5}{2}$. There is not a readily apparent triplet of states in the same energy region in ${ }^{77}$ As. However, van der Kooi and van den Bold ${ }^{7}$ ) proposed a level at $325 \mathrm{keV}$ on the basis of a gamma ray following the decay of ${ }^{77 \mathrm{~m}} \mathrm{Ge}\left(J^{\pi}=\frac{1}{2}^{-}\right)$. A gamma ray of $338.5 \mathrm{keV}$ following the decay of ${ }^{77} \mathrm{Ge}$ $\left(J^{\pi}=\frac{7}{2}^{+}\right)$has not been placed in our level scheme. This line might correspond to the ground state transition for such a level, and in combination with the $215.5\left(\frac{3}{2}^{-}\right)$

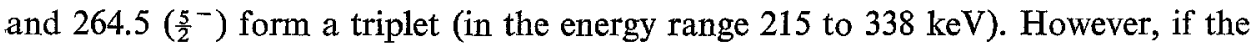
spin of such a level were $\frac{1}{2}$, the specific details of populating it are not clear.

Even though no nuclear model has as yet been able to predict in detail the measured properties of odd-mass nuclei in this region, a model which has received some attention for its ability to deal with the first few low-lying levels of such nuclei is the singleparticle core-coupling model ${ }^{12-15}$ ). In its simplest form the model describes the weak coupling of the single nucleon to the doubly even core. Here, the ground state would be ascribed to a coupling of the $p_{\frac{3}{2}}$ proton and the ground state of ${ }^{76} \mathrm{Ge}$, and four excited states with spins $\frac{1}{2}, \frac{3}{2}, \frac{5}{2}$, and $\frac{7}{2}$ would result from a coupling of the single proton to the $2^{+}$core state. One prediction obtained with the model is that the positions of these four levels will occur at excitation energies close to the energy of the $2^{+}$ level in the neighboring even-mass nucleus. In particular, the average energy of the quartet, each level being weighted according to its spin, should equal the energy of the ${ }^{76} \mathrm{Ge} 2^{+}$level. If the $\frac{1}{2}, \frac{3}{2}, \frac{5}{2}, \frac{7}{2}$ quartet is comprised of levels at $338.5,215.5,461.4$ and $784.8 \mathrm{keV}$, respectively, then the weighted energy of the multiplet is $549 \mathrm{keV}$. The energy ${ }^{16}$ ) of the $2^{+}$state in ${ }^{76} \mathrm{Ge}$ is $563 \mathrm{keV}$. This can be compared with the case of ${ }^{75}$ As where the spin weighted energy ${ }^{11}$ ) for the proposed multiplet was $573 \mathrm{keV}$; the energy of the $2^{+}$state in ${ }^{74} \mathrm{Ge}$ is $596 \mathrm{keV}$. Without some knowledge of reduced transition probabilities, further comparisons are limited. However, in cases where 
more experimental data are available (e.g. ref. ${ }^{11}$ ) for ${ }^{75} \mathrm{As}$ ), there are discrepancies between data and model predictions.

The authors would like to thank Dr. A. Bäcklin for sending us his computer program. One of us (D.D.) would like to thank Helmut Baer and Cecil Diethrich for their assistance and helpful comments.

\section{References}

1) J. R. Arnold and N. Sugarman, J. Chem. Phys. 15 (1947) 703

2) A. B. Smith, Phys. Rev. 86 (1952) 98

3) S. B. Burson, W. C. Jordon and J. M. LeBlanc, Phys. Rev. 96 (1954) 1555

4) A. W. Schardt, Phys. Rev. 108 (1957) 398

5) R. G. Arns, C. H. Chen, R. E. Sund and M. L. Wiedenbeck, Nucl. Phys. 40 (1963) 132

6) M. B. Martin and M. L. Wiedenbeck, Nucl. Phys. 48 (1963) 65

7) J. B. van der Kooi and H. J. van den Bold, Nucl. Phys. 70 (1965) 449

8) D. P. Donelly, H. W. Baer, J. J. Reidy and M. L. Wiedenbeck, Nucl. Instr. 57 (1967) 219

9) G. Murray, R. L. Graham and J. S. Geiger, Nucl. Phys. 63 (1965) 353

10) A. Bäcklin, Nucl. Instr. 53 (1967) 177 and private communication

11) R. L. Robinson, F. K. McGowan, P. H. Stelson and W. T. Milner, Nucl. Phys. A104 (1967) 401

12) A. de-Shalit, Phys. Rev. 122 (1961) 1530

13) M. Bouten and P. van Leuven, Nucl. Phys. 32 (1962) 499, 76 (1966) 479

14) H. E. Gove, Phys. Lett. 4 (1963) 249

15) V. K. Thankappan and W. W. True, Phys. Rev. 137 (1965) B793

16) Nuclear Data Sheets (National Research Council, Washington, D.C.) 